\title{
60. On the fundamental differential equations of flat projective geometry.
}

\author{
By Kentaro Yano. \\ Mathemarical Institute, Tokyo Imperial University. \\ (Comm. by S. KAKEYA, M.I.A., Oct. 12, 1945.)
}

$\S 1$. In an adress given at the International Mathematical Congress at Bolognil, ${ }^{1)}$ Prof. O. Vehlen defined the geometry as the theory of an invariant. According to the Erlanger Programm a geometry is the invariant theory of a group. According to the naw eonception of O. Veblen a geometry is the theory of invariant.

O. Veblen showed, in a lectur to the London Mathematical Society, ${ }^{2)}$ that the classical projective geometry mily be regarded as the theory of an invariant subject to curtiain restrictions. The theory of this invariant free from these restrictions is the so-called generalized projective geometry.

If we intruduce a curvilincar courdinate system $\left(\xi^{i}\right)^{3)}$ in an $n$-dimensional projective space, the homogeneous projective coodinates $Z^{\lambda}$ of the space may be expressed in the form

$$
Z^{\lambda}=e^{\xi \prime \prime} p_{\mu}^{\lambda} f^{\mu}\left(\xi^{i}\right)
$$

where $e^{\xi_{0}}$ is an arbitrary factor, $p_{\mu}^{\hat{\mu}}$ constants subject only to the condition that the determinant $p_{\mu .}^{\lambda}$ formed with $p_{\mu}^{\lambda}$ is defferent from zero and finally $f^{\lambda}(\xi)$ $n+1$ analytic functions of $\xi^{i}$ such that the detorminant

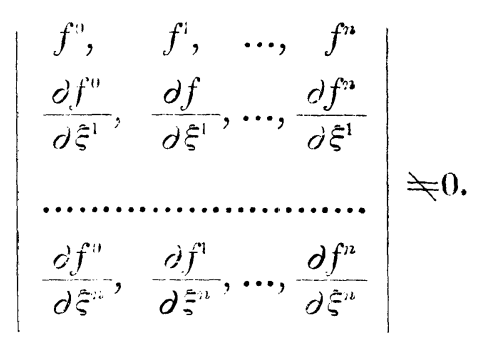

Differentiating (1.1) and eliminating the constants, we find that any $n+1$ homogencous projective coordincites $Z^{\lambda}$ defined as functions of curvilinear coordinates $\xi^{i}$ must satisfy the differential cquations

(1) O. Veblen: Differential invariants and geometry. Atti Congresso Internazionale Bolngna, 1, (1928), 181-189.

(2) O. Veblen: Generalized projective geometry. Journal of the London Math. Soc., 4 (1929), 140-160.

(3) Greek indices take the values on the range $0,1,2, \ldots, n$ and Roman indices on the range $1,2, \ldots, n$. 
No. 8.] On the fundamental differential equations of flat projective geometry.

$$
\frac{\partial^{2} Z}{\partial \xi^{\mu} \partial \xi^{\nu}}=\Gamma_{\mu \nu}^{\lambda} \frac{\partial Z}{\partial \xi^{\lambda}}
$$

where $\Gamma_{\mu \nu}^{\lambda}$ are functions of $\xi^{1}, \xi^{2}, \ldots, \xi^{n}$ only and satisfy the following conditions

$$
\begin{gathered}
(1.4) \\
(1.5) \\
\Gamma_{\omega \nu}^{\lambda}=\Gamma_{\nu 0}^{\lambda}=\delta_{\mu \nu}^{\lambda}, \\
(1.6) \quad B_{\mu \nu \nu \omega}^{\lambda} \equiv \Gamma_{\nu \mu,}^{\lambda}, \\
\end{gathered}
$$

The condition (1.4) follows from the special form of (1.1). The last two are integribility conditions of the differential equations (1.3).

The cquations (1.3) are the so-called fundamental differential equations of flat projective geometry.

As we may multiply the homogenecus projective coordinates by any factor and may change the curvilinear coordinate system to any other one, the equations (1.3) must be unaltered in the form by the transformations

$$
\left\{\begin{array}{l}
\overline{\xi^{\prime \prime}}=\xi^{\prime \prime}+\log \rho\left(\xi^{1}, \xi^{2}, \ldots, \xi^{n}\right), \\
\overline{\xi^{i}}=\xi^{i}\left(\xi^{1}, \xi^{2}, \ldots, \xi^{n}\right) .
\end{array}\right.
$$

By these transiormutiòns, the functions $\Gamma_{\mu \nu}^{\lambda}$ are transformed into $\bar{\Gamma}_{\mu \nu}^{\lambda}$, where

$$
\bar{\Gamma}_{\mu \nu}^{\lambda}=\frac{\partial \xi^{\lambda}}{\partial \xi^{\alpha}}\left(\frac{\partial \xi^{\beta}}{\partial \xi^{\mu}} \frac{\partial \xi^{\top}}{\partial \xi^{\nu}} \Gamma_{\beta \gamma}^{\alpha}+\frac{\partial^{2} \xi^{\alpha}}{\partial \bar{\xi}^{\mu} \partial \bar{\xi}^{\nu}}\right) \text {. }
$$

The conditions (1.4), (1.5) and (1.6) are invariant by these transformations.

As the transformation law (1.8) is transitive, we may define an invariant whose components in the coordinate system $\left(\xi^{\lambda}\right)$ are $\Gamma_{\mu \nu}^{\lambda}$ and whose components $\bar{\Gamma}_{\mu \nu}^{\lambda}$ in the coordinate system $\left(\bar{\xi}^{\lambda}\right)$ are given by $(1.8)$. This invariant is called the projective connection:

The theory of an arbitrary projective connection, whose components are functions of $n$ curcilinear coordinates and satisfy the conditions (1.4) and (1.5), is a generalized projective geometry. If the components of a projective connection satisfy the conditions (1.4), (1.5) and (1.6), the theory of this projective connection is flat projective geometry.

It is to be noticed that, if the components satisfy (1.4), (1.5) and (1.6), the components are functions of $\xi^{1}, \xi^{2}, \ldots, \xi^{n}$ only. In fact, we have, from (1.6),

$$
B_{\cdot \mu \nu 0}^{\lambda}=\frac{\partial \Gamma_{\mu \nu}^{\nu,}}{\partial \xi^{\prime \prime}}-\frac{\partial \Gamma_{\mu \prime \prime}^{\lambda}}{\partial \xi^{\nu}}+\Gamma_{\mu \nu}^{\alpha} \Gamma_{\alpha 0}^{\lambda}-\Gamma_{\mu 0}^{\alpha} \Gamma_{\alpha \nu}^{\lambda}=0
$$

from which 


$$
\begin{aligned}
& \partial I_{\mu \nu}^{\lambda}=0 . \\
& \partial \xi^{\prime}
\end{aligned}
$$

\$2. Let us consider (1.3) as the fundomeufal differential cquations of an $n+1$ dimensional affine space $A_{a+1}$ and $\Gamma_{\mu \nu}^{\lambda}$ as the components of affine coonection of the space.

If there exists, in $A_{n+1}$, a coordinate systom with repect to which the components $\Gamma_{\mu \nu}^{\lambda}$ of the affine connection satisfy the conditions (1.4), (1.5) and (1.6), then the affine space $A_{n+1}$, referred to this coordinate system, may be taken to represent the projective spacs $P_{n}$.

Following J. H. C. Whitehead, ${ }^{4)}$ we shall denote any coordinate system for $A_{n+1}$ by $K_{n+1}$ and a $K_{n+1}$ which is a representation for $P_{n}$ by $R_{n}$.

Let

$$
Z^{\lambda}=e^{\xi^{0}} f^{\lambda}\left(\xi^{i}\right)
$$

be a set of $n+1$ independent solutions of the differential equations. The fret that the solutions are of the form (2.1) is ensured by the conditions (1.4). If we consider the equations (1.3) as the fundamental difierential equations of the $(n+1)$-dimensional flat affine space $A_{n+1}$ and $\Gamma_{\mu \nu}^{\lambda}$ as the components of the alfine connection, the solutions $Z^{\lambda}$ given by (2.1) are rectilinear affine coodinates of the space, and with respect to this coordinate system, all the components of the afffine connection vanish.

Thus, the rectilinear coordinate system $\left(Z^{\lambda}\right)$ is an $K_{n+1}$ for the affine spisce, but it is not a representation for the proinctive spece $P_{: \iota}$, that is, is is not an $\boldsymbol{P}_{u, 2}$. This is ciue to the fact that we are using the non-homogeneous coordinate system but not the homogeneous one.

To obtain an $R_{\imath \iota}$, we put

$$
\left\{\begin{array}{l}
z^{0}=\log Z^{\prime \prime} \\
\varkappa^{i}=Z^{i} \mid Z^{\prime \prime}
\end{array}\right.
$$

or

$$
\left\{\begin{array}{l}
z^{0}=\xi^{n}+\log f^{n}\left(\xi^{1}, \xi^{2}, \ldots, \xi^{i n}\right), \\
z^{i}=f^{i}\left(\xi^{j}\right) .
\end{array}\right.
$$

The transformation of coordinates $\xi^{\lambda} \rightarrow z^{\lambda}$ is of the form (1.7). If we denote by $P_{\mu \nu}^{\lambda}$ the components of the prujective comnection, we have, as it maty be easily verified,

$$
P_{i,}^{\lambda}=P_{\nu_{0}}^{\lambda}=\delta_{j}^{\lambda} \text { and } P_{j k}^{\lambda}=0
$$

4) J.H.C. Whitehead: On a class of projectively flat affine connections. Proc. London Math. Soc., 32 (1931), 93-114; The representation of projective spaces. Annals of Math., 32 (1931), 327-360. 
No. 3.] On the fundamental differential equations of flat projective geometry.

Thus the coordinate system $\left(z^{\lambda}\right)$ is an $\boldsymbol{R}_{\imath *}$.

If we regard $Z^{\lambda}$ as a rectilinear coorclinetes in $A_{n+1}$, the relation between $\left(Z^{\lambda}\right)$ and $\left(z^{x}\right)$ is given by

$$
\left\{\begin{array}{l}
Z^{0}=e^{z ?}, \\
Z^{i}=e^{z 0} z^{i} .
\end{array}\right.
$$

Thus the points of $P_{n:}$, which are represented, in $A_{n+1}$, by the straight lines through the origin, are represented, in $\left(z^{\lambda}\right)$, by the curves of parametres $z^{0}$.

$\S 3$. In the flat case, the finite equations of the paths, say, of the straight lines are given by

$$
Z^{\lambda}=A^{\lambda}+B^{\lambda} t
$$

where $A^{\lambda}$ and $B^{\lambda}$ are homogeneous coordinates of two points on the straight line. Differentiating this equation: twice with respect to $t$ and taking account of the fundimental differential equations, we find

$$
\frac{d^{2} \xi^{\lambda}}{d t^{2}}+\Gamma_{\mu \nu}^{\lambda} \frac{d \xi^{\mu}}{d t} \frac{d \xi^{\nu}}{d t}=0
$$

as the differential cquations of the paths.

Putting $\lambda=0$ and $\lambda=i$ respectively in (3.2), we have

$$
\left\{\begin{array}{l}
\text { (i ) } \frac{d^{2} \xi^{0}}{d t^{2}}+\left(\frac{d \xi^{0}}{d t}\right)^{2}+\Gamma_{j k}^{n} \frac{d \xi^{j}}{d t} \frac{d \xi^{k}}{d t}=0, \\
\text { (ii) } \frac{d^{2} \xi^{i}}{d t^{2}}+\Gamma_{j k}^{i} \frac{d \xi^{j}}{d t} \frac{d \xi^{k}}{d t}+2 \frac{d \xi^{0}}{d t} \frac{d \xi^{i}}{d t}=0
\end{array}\right.
$$

in virtue of the relations $\Gamma_{0 \nu}^{\lambda}=\Gamma_{\nu 0}^{\lambda}=\delta_{\nu}^{\lambda}$. If we put

$$
2 \frac{d \xi^{0}}{d t}=\frac{d^{2} t}{d s^{2}} /\left(\frac{d t}{d s}\right)^{2}
$$

or

$$
\xi=\frac{1}{2} \log \frac{d t}{d s},
$$

we have, from (3.3) (i),

$$
\{t, s\}=-2 \Gamma_{j k}^{0} \frac{d \xi^{j}}{d s} \frac{d \xi^{k}}{d s},
$$

and, from (3.3) (ii),

$$
\frac{d^{2} \xi^{i}}{d s^{2}}+\Gamma_{j k}^{i} \frac{d \xi^{j}}{d s} \frac{d \xi^{k}}{d s}=0
$$

where $\{t, s\}$ is the Schwarzian derivative of $t$ with respect to $s, s$ being : an affine parameter on paths. Thus the parameter $t$ is a projective one.

If we take the coordinate system $\left(z^{\lambda}\right)$ defined in the last Paragraph, the equations (3.5) and (3.6) gives 


$$
t=\frac{a s+\beta}{\gamma s+\delta}
$$

and

$$
z^{i}=a^{i} s+b^{i}
$$

respectively, where $\alpha, \beta, \gamma, \delta, a^{i}$ and $b^{i}$ are constants.

The parameter $t$ introduced in the equation of path (3.1) is a projective one. If we use an arbitrary parameter $r$ on path, the finite equation of the paths is

$$
Z^{\lambda}=A^{\lambda} u(r)+B^{\lambda} v(r) \text {. }
$$

Differentiating twice this equation with respect to $r$, and eliminating constants from these equations, we find the differential equations of the form

$$
\frac{d^{2} Z^{\lambda}}{d r^{2}}=\alpha \frac{d Z^{\lambda}}{d r}+\beta Z^{\lambda}
$$

The homogeous coordinates $Z^{\lambda}$ being functions of curvilinear coordinates $\xi^{\lambda}$, we have, from (3.10),

$$
\frac{d^{2} \xi^{\lambda}}{d r^{2}}+\Gamma_{\mu \nu}^{\lambda} \frac{d \xi^{\mu}}{d r} \frac{d \xi^{\nu}}{d r}=\alpha \frac{d \xi^{\lambda}}{d r}+\beta \delta_{0}^{\lambda}
$$

in virtue of the fundamental differential equations and of the relation $Z^{\lambda}=\frac{\partial Z^{\lambda}}{\partial \xi^{0}}$.

The equations (3.11) are the most general equations of the paths.

If we regard $Z^{\lambda}$ or $\xi^{\lambda}$ as the-coordinates of points in an $(n+1)$-dimensional affine space $A_{n+1}$, the equations (3.1) are those of straight lines passing by two points $A^{\lambda}$ and $B^{\lambda}$. The points $A^{\lambda}$ and $B^{\lambda}$ of projective space $P_{n}$ being represented by a straight lines in $A_{n+1}$ joining these points to origin, we may say that the paths in $P_{n}$ are represented by a plane in $A_{n+1}$ containing three points $A^{\lambda}, B^{\lambda}$ and the origin.

Thus, the paths in $P_{n}$ being represented by planes passing through the origin in $A_{n+1}$, to represent the paths, we may take arbitrary curres on these planes passing through the origin. The equations (3.11) are equations of such eurves. Indeed, the equations show that, if we regurl $\xi^{\lambda}$ as coordinate of points in an $(n+1)$-dimonsional affine space $A_{n+1}$, the osculating plane of this curve contains always the vector $\delta_{0}^{\lambda}$. The vector whose components are $\delta_{0}^{\lambda}$ being tangent to the $\xi^{0}$-curves or rays, it passes through always the origin.

$\S 4$. In Paragraph 1, we have assumed the homogeneous projective coordinates $Z^{\lambda}$ of an $n$-dimensional projective space to be of the form (1.1), $\xi^{\prime}$ and $\xi^{i}$ being an arbitrary factor and curvilinear coordinates of the space respectively.

Let us now introduce, in an n-dimensional projective space $P_{n}$, a system of 
No. 8.] On the fundamental differential equations of flat projective geometry.

curvilinear homogeneous coordinates $\left(x^{2}\right)$ and assume that the homogeneous projective coordinates $Z^{\lambda}$ of the space are expressed in the form

$$
Z^{\lambda}=x_{\mu}^{\lambda} F^{\mu}\left(x^{\alpha}\right)
$$

where $p_{\mu}^{\lambda}$ are constants subject only to the conditions that the determinant $\left|p_{\mu}^{\lambda}\right|$ formed with them is different from zero and $F^{\lambda}\left(x^{\alpha}\right)$ are $n+1$ homogeneous functions of degree +1 such that

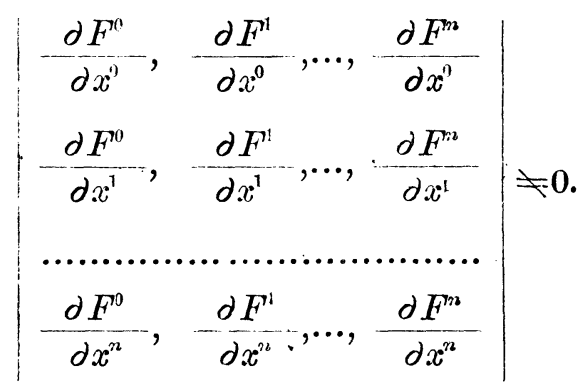

Differentiating (4.1) and eliminating the constants $p_{\mu}^{\lambda}$, we find the so-called fundamental differential fquations

$$
\frac{\partial^{2} Z}{\partial x^{\mu} \partial x^{\nu}}=I_{\mu \nu}^{\lambda} \frac{\partial Z}{\partial x^{\lambda}}
$$

which any $n+1$ homogeneous projective coordinates $Z^{\lambda}$ defined as the functions of $n+1$ curvilinear homogeneous coordinates $x^{\lambda}$ must satisfy.

The functions $Z^{\lambda}$ being homogeneous of degree +1 with respect to $x^{\alpha}$, the coefficients $I_{\mu \nu}^{\lambda}$ are homogeneous tunctions of the degree $-\mathbf{1}$ with respect to $x^{\alpha}$.

The functions $Z^{\lambda}$ being homogeneous of degree +1 , the functions $\frac{\partial Z}{\partial x^{\lambda}}$ are homogeneous of degree 0 , so that if we multiply (4.3) by $x^{\lambda}$ and sum up for the index $\nu$, we find

$$
0=I_{\mu \nu}^{\lambda} x^{\nu} \frac{\partial Z}{\partial x^{\lambda}}
$$

from which we obtain

$$
I I_{\mu \nu}^{\lambda} x^{\nu}=0
$$

the $n+1$ points being linearly independent in virtue of (4.2).

The integrability conditions of the differential equations (4.3) are

$$
\begin{gathered}
\Pi_{\mu \nu}^{\lambda}=\Pi_{\nu \mu}^{\lambda}, \\
\Omega_{\cdot \mu \nu \omega}^{\lambda} \equiv \frac{i \Pi_{\mu \nu}^{\lambda}}{\partial x^{\omega \nu}}-\frac{\partial \Pi_{\mu \omega \nu}^{\lambda}}{\partial x^{\nu}}+I I_{\mu \nu}^{\alpha} I_{\alpha, i \nu}^{\lambda}-I I_{\mu \omega}^{\alpha} I I_{\alpha \nu}^{\lambda}=0 .
\end{gathered}
$$

As we may change the curvilinear homogeneous coordinates to any other one, the equations (4.3) must be unaltered in the form by the transformation

$$
x^{\lambda}=x^{\lambda}\left(x^{n}, x^{1}, \ldots, x^{n}\right),
$$


where $a^{2}(x)$ are homogeneous functions of degree one with respect to their arguments.

By these trinsiormations, the functions $I_{\mu \nu}^{\lambda}$ are transformed into $I_{\mu \nu}^{\lambda}$ following the formulae of the same form as (1.8),

$$
\bar{I}_{\mu \nu}^{\lambda}=\frac{\partial \bar{x}^{\lambda}}{\partial x^{\alpha}}\left(\frac{\partial x^{\beta}}{\partial x^{\mu}} \frac{\partial x^{\gamma}}{\partial x^{\nu}} I_{\beta \gamma}^{\alpha}+\frac{\partial^{2} x^{\alpha}}{\partial x^{\mu} \partial x^{\nu}}\right) \text {. }
$$

The conditions (4.5), (4.6) and (4.7) are invariant by these transformmations.

As we may multiply homogencous projective cuordinates by any factor, the equations (4.3) must be invariant also by the transformation

$$
{ }^{\prime} Z^{\lambda}=\sigma Z^{\lambda} \quad \text { or } \quad x^{\lambda}=\sigma x^{\lambda}
$$

where the factor $\sigma(x)$ is a lomogereous function of degree zero with respect to the $: x^{\lambda}$.

It will be easily seen that, by this transformation, the functions $I_{\mu \nu}^{\lambda}$ are transformed into ' $\Pi_{\mu \nu}^{\lambda}$ following the formulae

$$
' I I_{\mu \nu}^{\lambda}=I I_{\mu \nu}^{\lambda}+\delta_{\mu \nu}^{\lambda} \sigma_{\nu}+\delta_{\nu}^{\lambda} \sigma_{\mu}+\sigma_{\mu \nu} x^{\lambda},
$$

where

$$
\sigma_{\nu}=\frac{\partial \log \sigma}{\partial x^{\nu}} \text { and } \quad \sigma_{\mu \nu}=\frac{\partial \sigma_{\mu}}{\partial x^{\nu}}-\sigma_{\lambda} \Pi_{\mu \nu}^{\lambda}-\sigma_{\mu} \sigma_{\nu}
$$

The conditions (4.5), (4.6) and (4.7) are also invariant under such transformation of coordinates.

As the transformation laws (4.9) and (4.11) of $\Pi_{\mu \nu}^{\lambda}$ are transitive, we may define, as in Paragraph 1, an invariant whose compononts in the coordinate system $\left(x^{\alpha}\right)$ are $\Pi_{i \nu \nu}^{\lambda}$ and whose components $\bar{\Pi}_{\mu \nu}^{\lambda}$ and ' $\Pi_{\mu \nu}^{\lambda}$ in other coordinate systems $\left(\bar{x}^{\alpha}\right)$ and $\left({ }^{\prime} x^{\alpha}\right)$ are given by (4.9) and (4.11) respectively. This invariant may be called the projective connection.

Thus, a generalized projective geometry ${ }^{55}$ may be defined as the theory of an arbitrary projective connection, whose components are functions of $n+1$ curvilinear homogeneous coordinates and satisfy the conditions (4.5) and (4.6). If the components of a projective connection satisfy the conditions (4.5), (4.6) and (4.7), the theory of this projective connection is flat projective geometry.

It is to be noticed that, if the components satisfy (4.5), (4.6) and (4.7), these components are homogeneous functions of degree -1 with respect to $x^{x}$. In fact, we have, from (4.7)

5) J. A. Schouten and J. Haantjes: Zur allgemeinen projektıven Differentialgeometrie, Comp. Matb., 3 (1.936), 1-51. 
No. 8.] On the fundamental differential equations of flat projective geometry.

$$
\Omega_{. \mu \nu \omega}^{\lambda} x^{\omega}=\left(\frac{\partial \Pi_{\mu \nu}^{\lambda}}{\partial x^{\omega}}-\frac{\partial I_{\mu \omega}^{\lambda}}{\partial x^{\nu}}+\Pi_{\mu \nu}^{\alpha} I_{\alpha \omega}^{\lambda}-I I_{\mu \omega}^{\alpha} I_{\alpha \nu}^{\lambda}\right) x^{\omega}=0
$$

from which

$$
\frac{\partial I_{\mu \nu}^{\lambda}}{\partial x^{\omega}} x^{\omega}=-I_{\mu \nu}^{\lambda}
$$

$\S 5$. In this Paragraph; we shall study the relations between two components of the projective connection $\Gamma_{\mu \nu}^{\lambda}$ and $I_{\mu \nu}^{\lambda}$, one introduced in $\S 1$ and the other in the last Paragraph.

The non-homogeneous curvilinear coordinates $\xi^{i}$ and the homogeneous curvilinear coordinates $x^{\lambda}$ being both coordinate systems for an $n$-dimensional projective space $P_{n}$, we must have the equations of the form

$$
\left\{\begin{array}{l}
\xi^{\alpha}=\log \rho\left(x^{\alpha}\right), \\
\xi^{i}=\xi^{i}\left(x^{\alpha}\right)
\end{array}\right.
$$

between these coordinates where the functions $\xi^{0}\left(x^{\alpha}\right)$ and $\xi^{\alpha}\left(x^{\alpha}\right)$ are homogeneous of degree one and zero respectively with respect to $x^{\alpha}$. Thus, if we put

$$
\frac{\partial \log \rho}{\partial x^{\lambda}}=\rho_{\lambda} \quad \text { and } \quad E_{\cdot \lambda}^{i}=\frac{\partial \xi^{i}}{\partial x^{\lambda}}
$$

we have

$$
\rho_{\lambda} x^{\lambda}=1 \text { and } E_{\cdot 2}^{i} x^{\lambda}=0
$$

As we have

$$
\begin{gathered}
\frac{\partial Z}{\partial x^{\mu}}=\rho_{\mu} Z+E_{\cdot \mu}^{i} \frac{\partial Z}{\partial \xi^{j}} \\
\frac{\partial^{2} Z}{\partial x^{\mu} \partial x^{\nu}}=\frac{\partial \rho_{\mu}}{\partial x^{\nu}} Z+\rho_{\mu} \rho_{\nu} Z+E_{\cdot \mu}^{j} \rho_{\nu} \frac{\partial Z}{\partial \xi^{j}}+E_{. \nu}^{j} \rho_{\mu} \frac{\partial Z}{\partial \xi^{j}} \\
+E_{\circ \mu}^{j} E_{\cdot \nu}^{i} \frac{\partial^{2} Z}{\partial \xi^{j} \partial \xi^{k}}+\frac{\partial E_{\cdot \mu}^{i}}{\partial x^{\nu}} \frac{\partial Z}{\partial \xi^{i}}
\end{gathered}
$$

substituting the fundamental equations (1.3) and (4.3) in (5.4), we have

$$
\begin{aligned}
& \rho_{\lambda} \Pi_{\mu \nu}^{\lambda} Z+E_{\cdot \lambda}^{i} \Pi_{\mu \nu}^{\lambda} \frac{\partial Z}{\partial \xi^{i}} \\
& \quad=\frac{\partial \rho_{\mu}}{\partial x^{\nu}} Z+\rho_{\mu} \rho_{\nu} Z+E_{\cdot \mu}^{j} \rho_{\nu} \frac{\partial Z}{\partial \xi^{j}}+E_{\cdot \nu}^{j} \rho_{\mu} \frac{\partial Z}{\partial \xi^{j}}+E_{\cdot \mu}^{j} E_{\cdot \nu}^{k} \Gamma_{j k}^{0} Z \\
& \quad+E_{\cdot \mu}^{j} E_{\cdot \nu}^{k} \Gamma_{j k}^{i} \frac{\partial Z}{\partial \xi^{i}}+\frac{\partial E_{\cdot \mu}^{i}}{\partial x^{\nu}} \frac{\partial Z}{\partial \xi^{i}}
\end{aligned}
$$

in virtue of the equation (3.5).

The $n+1$ points $Z$ and $\frac{\partial Z}{\partial \xi^{i}}$ being independent, we have from the above equation 


$$
E_{\cdot \mu}^{j} E_{\cdot \nu}^{k} \Gamma_{j k}^{0}=-\left(\frac{\partial \rho_{\mu}}{\partial x^{\nu}}-\rho_{\lambda} I I_{\mu \nu}^{\lambda}-\rho_{\mu \nu} \rho_{\nu}\right)
$$

and

$$
E_{* \mu}^{i} E_{* \nu}^{k} \Gamma_{j k}^{i}=-\left(\frac{\partial E_{* \mu}^{i}}{\partial x^{\nu}}-E_{* \lambda}^{i} I_{\mu \nu}^{\lambda}+E_{\cdot \mu}^{i} \rho_{\nu}+E_{\bullet \cdot \rho_{\mu \nu}}^{i}\right)
$$

If we denote by

$$
\left(\begin{array}{l}
x^{\lambda} \\
E_{i}^{* \lambda}
\end{array}\right)^{\prime}
$$

the inverse metrix of

$$
\left(\begin{array}{l}
\rho_{\lambda} \\
E_{\cdot \lambda}^{i}
\end{array}\right)
$$

we can solve the equations (5.5) and (5.6) with respect to $\Gamma_{j k}^{0}$ and $I_{j k}^{i}$ respectively, say,

$$
\begin{gathered}
\Gamma_{j k}^{\prime \prime}=-E_{j}^{\mu \nu} E_{k}^{\nu}\left(\frac{\partial \rho_{\mu \mu}}{\partial x^{\nu}}-\rho_{\lambda} I I_{\mu \nu}^{\lambda}\right), \\
\Gamma_{j k}^{i}=-E_{j}^{\mu \mu} E_{k}^{\nu \nu}\left(\frac{\partial E_{\cdot \mu}^{i}}{\partial x^{\nu}}-E_{\cdot \lambda}^{i} I_{\mu \nu}^{\lambda}\right) .
\end{gathered}
$$

\$6. In this last Paragraph, we shall investigate the differential equations of paths in the case where we are using the curvilinear homogeneous coordinates. The equation of paths being

$$
Z^{\lambda}=A^{\lambda} u(r)+B^{\lambda} v(r)
$$

we have the differential equations

$$
\frac{d^{2} Z^{\lambda}}{d r^{2}}=\alpha \frac{d Z^{\lambda}}{d r}+\beta Z^{\lambda}
$$

from which, we find

$$
\frac{d^{2} x^{\lambda}}{d r^{2}}+I_{\mu \nu}^{\lambda} \frac{d x^{\mu}}{d r} \frac{d x^{\nu}}{d r}=\alpha-\frac{d x^{\lambda}}{d r}+\beta x^{\lambda}
$$

by virtue of the fund:mental equations.

As we have already stated, these equations give in an $(n+1)$-dimensional represcntation, a curve whose osculating plane passes always by the origin and hence a curve on a plane passing through the origin.

Thus the transformation (4.11) may be called the subprojective transformation $^{6)}$ of the affine connection.

6) K. Yano: Subprojective transformations, subprojective spaces and subprojective collineations. Proc., 20 (1944), 701-705. 